\section{Capillary blood flow in the diabetic neuropathic foot}

Dear Sir

In their article on the measurement of capillary blood flow in the diabetic neuropathic foot Flynn et al. [1] claimed to have measured the nutritive capillary component of the circulation. They considered that their results refuted the possibility of a role for rheological factors in capillary closure.

Because their measurements were based on atypically large capillaries in a tissue which is involved only rarely in ulceration it seems that the general application of their results are unsustainable. If the authors claim to refute the role of abnormal blood rheology in ulcerogenesis, then it seems to be important that their measurements have rheological relevance, and this was not the case. As the diameter of the capillaries studied was much larger than the diameter of erythrocytes it is unlikely that the intrinsic stiffening of the diabetic erythrocyte membrane would have the same effects on blood flow that it would have in smaller capillaries.

It seems strange that ulceration should develop in tissue where capillary blood flow was significantly increased. The authors extrapolated from their nail-fold measurements to claim that "... footskin capillary blood flow is increased in diabetic patients." Increased flow rates of blood in capillaries is unlikely to occur without a rise in intracapillary pressure and a resulting increase in transudation, although these aspects were not discussed.

If the primary lesion which develops into an ulcer is not based upon an ischaemic episode (most likely involving the well-confirmed haemorheologic abnormalities of diabetic blood) could the authors be asked to explain how they envisage ulceration developing in well oxygenated tissue? Do the authors believe that data relating to blood flow in nailfold capillaries provides meaningful information about blood flow in malleolar skin capillaries?

\section{O.Simpson}

\section{References}

1. Flynn MD, Edmonds ME, Tooke JE, Watkins PJ (1988) Direct measurement of capillary blood flow in the diabetic neuropathic foot. Diabetologia 31: 652-656

Dr. L.O. Simpson

Department of Pathology

Medical School

P.O. Box 913

Dunedin

New Zealand

\section{Response from the authors}

Dear Sir,

The aim of our recent paper [1] was to measure for the first time the nutritional capillary component of skin blood flow in the feet of diabetic patients with and without neuropathy. The stimulus to this study was that previous workers had postulated the coexistence of increased arterio-venous shunt flow with reduced capillary flow producing skin ischaemia. It had been proposed that either the high arterio-venous shunt flow led to a "capillary steal syndrome" or that the capillaries were occluded by "advanced micro-angiopathy", diverting blood proximally through arterio-venous shunts.

Television microscopy is the only method available to directly measure capillary flow. Capillary flow can only be measured in the nail-fold capillaries and whilst these are slightly larger than some other capillaries it has not been demonstrated that these are functionally different. Nail-fold capillaries are distal to arterio-venous shunts. Thus, with the above qualification we believe our results can be extrapolated to the plantar area of the foot. This is the area most commonly involved in neuropathic ulceration, rather than the malleolus.

In our study we report our observation of similar numbers of flowing capillaries in diabetic patients with and without neuropathy compared to normal control subjects. Thus, in the neuropathic foot under the conditions of study we saw no evidence of capillary closure. We state clearly that we could not exclude the possibility that tissue breakdown and ulceration are finally precipitated by acute capillary closure and thus by implication abnormal blood rheology and other factors may of course have a role in ulcer formation.

In our study we examined capillary blood flow at rest. We speculate that the increase in skin blood flow may be insufficient to meet the increased metabolic needs of the skin at the higher temperatures of the diabetic neuropathic foot. It is also recognised that the ability of the diabetic micro-circulation to increase perfusion in response to injury is limited [2]. Thus, the increase in the resting blood flow is compatible with the potential for ulceration.

We did not discuss changes in intracapillary pressure and fluid transudation. We observed that there was an increase in tissue oedema during our study but were unable to quantitate this in any suitable way. Measurements of capillary pressure in diabetic patients have been reported elsewhere [3].

We therefore believe that our report of the direct measurement of capillary flow in the toe nail-fold is valid and provides meaningful information about blood flow in the diabetic patient. Tissue ulceration in the insensitive diabetic foot is the ultimate consequence of numerous abnormalities including abnormal theology. The terminal event must be the failure of the microcirculation to deliver adequate oxygen to the skin, whatever the underlying mechanism.

Yours faithfully,

M.D. Flynn, M.E.Edmonds, J.E.Tooke, P.J.Watkins

\section{References}

1. Flynn MD, Edmonds ME, Tooke JE, Watkins PJ (1988) Direct measurements of capillary blood flow in the diabetic neuropathic foot. Diabetologia 31: 652-656

2. Rayman G, Williams SA, Spencer PD, Smafe LH, Wise PH, Tooke JE (1986) Impaired microvascular response to minor skin trauma in type 1 diabetes. Br Med J 292: 1295-1298

3. Tooke JE (1980) Capillary pressure disturbances in young diabetics. Diabetes 29: $815-819$

Dr. M. D. Flynn

Medical Unit, Torridge Ward, Level 8

Royal Devon and Exeter Hospital

Barrack Road

Exeter EX2 5DW

UK 